\title{
Tunicamycin potently inhibits tumor necrosis factor-induced hepatocyte apoptosis
}

\author{
Marcel Leist, Albrecht Wendel * \\ Biochemical Pharmacology, Faculty of Biology, University of Konstanz, POB 5560-M668, D-78434 Konstanz. Germany
}

\begin{abstract}
The protein glycosylation inhibitor tunicamycin protected male BALB/c mice from tumor necrosis factor $\alpha$-induced liver failure. Tunicamycin also inhibited tumor necrosis factor-induced cell death in primary hepatocyte cultures with a median inhibitory concentration of $8 \mathrm{nM}$, but not in the tumor cell line WEHI 164 clone 13. Hepatocyte death in our culture system was characterized by DNA fragmentation and apoptotic changes. These two characteristic signs of programmed cell death were also inhibited by tunicamycin treatment. These data suggest that protein glycosylation is an early and causal event of tumor necrosis factor (TNF)-induced parenchymal cell death in the liver.
\end{abstract}

Keywords: Septic liver failure: Glycosylation inhibitor: Liver, mouse; DNA fragmentation

\section{Introduction}

Tumor necrosis factor (TNF) is a cytokine with a vast spectrum of physiological and pathophysiological functions characterized initially by its tumor-necrotizing and cachexia-inducing properties. TNF is released from tissue macrophages or various blood leukocytes during inflammatory reactions. At high systemic concentrations it may cause circulatory and multi organ failure in animals and man (Tracey and Cerami, 1993; Waage, 1992). Hepatotoxicity due to TNF may become manifest in clinical systemic inflammatory response syndromes and also represents a major restriction of the chemotherapeutic use of the cytokine. In vitro, TNF was shown to induce programmed cell death in murine hepatocytes under the condition of transcriptional arrest. However, in these primary liver cell cultures intact translation was required for the induction of apoptosis by TNF (Leist et al., 1994).

Tunicamycin inhibits $\mathrm{N}$-linked protein glycosylation by blocking the transfer of $\mathrm{N}$-acetylglucosamine-1phosphate from UDP- $N$-acetylglucosamine to dolichyl

\footnotetext{
* Corresponding author. Tel.: +49-7531-882113; Fax: +49-7531883099.
}

phosphate (Elbein, 1981). It consists of variously acylated homologs of a conserved uridine-containing trisaccharide structure (Duksin and Mahoney, 1982). Tunicamycin does not directly affect the synthesis of the polypeptide chain of proteins. Attempts have been made to use the compound as an experimental chemotherapeutic agent. since it has been reported to be selectively cytotoxic towards transformed cell lines (Kohno et al., 1979). In the present study we describe the protective action of this antibiotic against TNF-induced cell death in untransformed murine hepatocytes in vitro and its protective properties against TNF-induced hepatotoxicity in mice.

\section{Materials and methods}

\subsection{Reagents}

Recombinant murine TNF- $\alpha$ was generously provided by Dr. Adolf, Boehringer Institute (Vienna, Austria). Cell culture media were from Biochrom (Berlin, Germany). GalN was purchased from Roth (Karlsruhe, Germany). Tunicamycin was from Boehringer (Mannheim, Germany), collagenase. actinomycin D and all other substances were purchased from Sigma (Deisenhofen, Germany). 


\subsection{Animal experiments}

Male BALB/c mice ( $25 \mathrm{~g}$ ) were from the internal animal breeding house, University of Konstanz. For cell preparations mice were fed ad libitum, whereas they were starved overnight for the in vivo experiments. All animals received humane care in accordance with the recommendations of the Helsinki declaration and Germany's legal requirements.

At 8 a.m. animals were injected in the tail vein with TNF $(3.3 \mu \mathrm{g} / \mathrm{kg}$ in a volume of $300 \mu \mathrm{l}$ saline containing $1 \% \mathrm{BSA}$ ). Liver damage was assessed by measuring plasma enzymes $8 \mathrm{~h}$ later according to Bergmeyer (1984). Actinomycin D or tunicamycin were given intraperitoneally in a volume of $200 \mu \mathrm{I}$ saline, $2 \mathrm{~min}$ or 30 min, respectively, before TNF injection. Blood was sampled in heparin-coated syringes by cardiac puncture immediately after killing the animals by cervical dislocation.

\subsection{Cell cultures}

WEHI-164 clone 13 cells were used for the TNF toxicity determination essentially as described by Espevik and Nissen-Meyer (1986). Hepatocytes were isolated by the two step collagenase perfusion method of
Seglen (1973) with a viability exceeding $80 \%$ according to the trypan blue exclusion method. Cells were plated in $200 \mu \mathrm{l}$ RPMI 1640 medium containing $10 \%$ newborn calf serum in 24-well plates at a number of $8 \times 10^{4}$ hepatocytes per well. They were allowed to adhere to culture plates for $5 \mathrm{~h}$ before the medium was exchanged for RPMI 1640 medium without serum. Experiments were carried out in an incubator supplied with $5 \% \mathrm{CO}_{2}, 40 \% \mathrm{O}_{2}$ and $55 \% \mathrm{~N}_{2}$ for the times indicated.

\subsection{Cytotoxicity, chromatin condensation and DNA fragmentation}

Lactate dehydrogenase was determined (Bergmeyer, 1984 ) in culture supernatants (S), and in the remaining cell monolayer (C) after lysis with Triton X-100. The percentage of lactate dehydrogenase release was calculated from the ratio of $S /(S+C)$. DNA fragmentation was determined by agarose gel electrophoresis (Wyllie. 1980). Fluorescence microscopy was performed on an Axiovert 35, Carl Zeiss (Oberkochen, Germany) using hepatocytes fixed for $10 \mathrm{~min}$ in $3.7 \%$ formaldehyde and stained for $15 \mathrm{~s}$ with $8 \mu \mathrm{g} / \mathrm{ml}$ bisbenzimide H33258. Riedel-de Haen (Seelze, Germany).
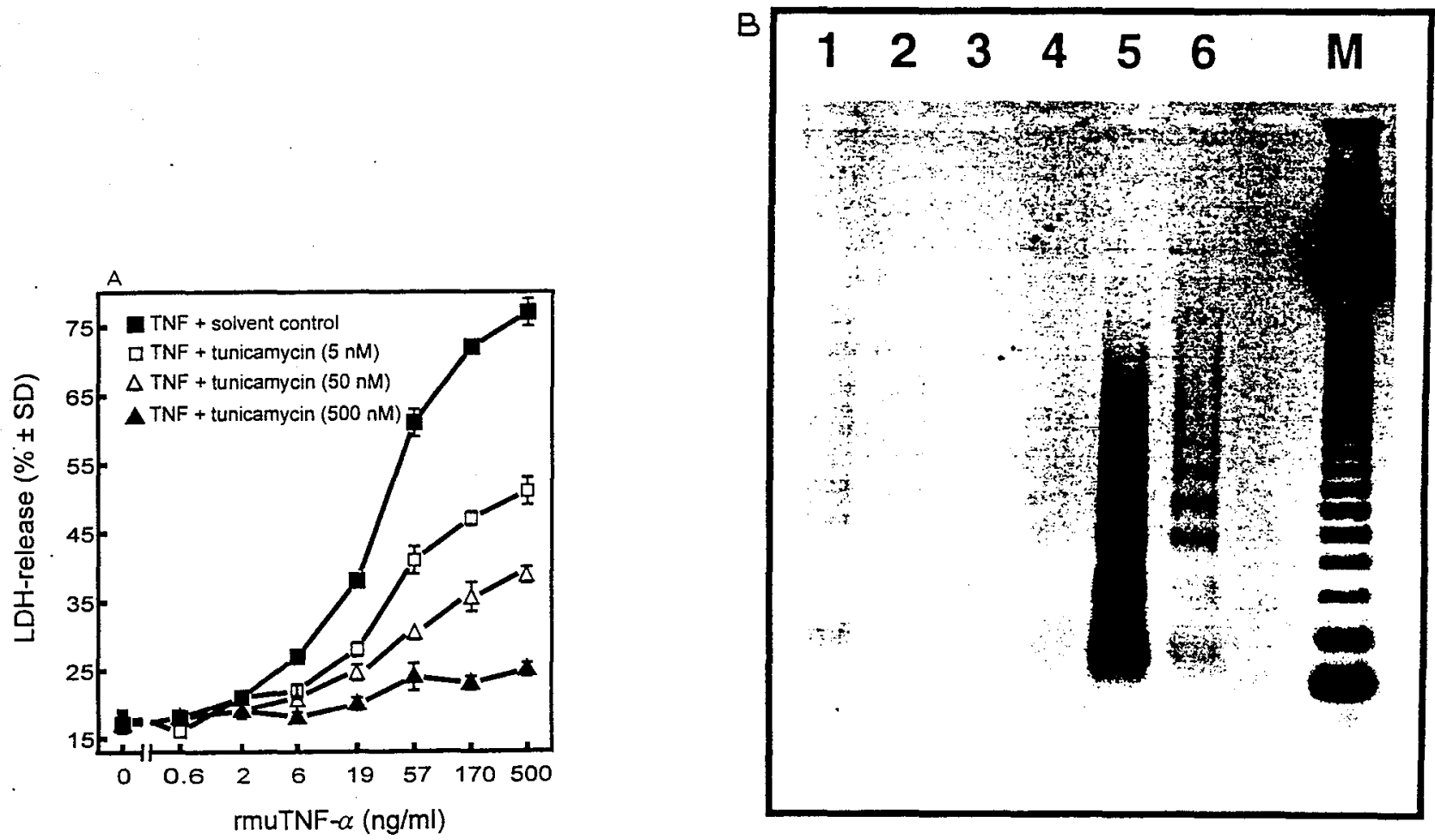

Fig. 1. Protection by tunicamycin of primary murine hepatocytes from actinomycin D/TNF-induced apoptosis. $6 \mathrm{~h}$ after cell isolation incubations were initiated by medium change and addition of tunicamycin and actinomycin D (333 nM). TNF was added 30 min later. $A$ : toxicity was determined after $18 \mathrm{~h}$ by quantitation of the lactate dehydrogenase (LDH) release. Data are means $\pm \mathrm{SD} . B: 10 \mathrm{~h}$ after TNF addition cytosolic low molecular DNA was prepared and analysed by electrophoresis on a $1 \%$ agarose gel. M: molecular weight marker ( $n$ times 123 base pairs); 1 : actinomycin D (333 nM); 2: TNF (100 ng/ml); 3: no cells; 4: solvent control; 5: actinomycin D/TNF; 6: actinomycin D/TNF plus $500 \mathrm{nM}$ tunicamycin. 


\section{Results}

\subsection{Protection by tunicamycin against TNF-induced liver failure in actinomycin D-sensitized mice}

In order to extend our previous findings on the protection by inhibitors of protein synthesis against TNF-induced septic liver failure (Leist et al., 1994), we used the glycosylation inhibitor tunicamycin in order to examine whether glycoprotein synthesis is required for the manifestation of TNF-induced hepatotoxicity. The data in Table 1 show that actinomycin D/TNF-induced liver injury was significantly reduced in mice that had been pretreated with tunicamycin. Control experiments showed that actinomycin D, TNF or tunicamycin alone did not significantly affect the plasma enzyme activities measured $8 \mathrm{~h}$ after administration of these agents. All animals treated with actinomycin D/TNF died within $8 \mathrm{~h}$, whereas no mortality was observed within this time span in mice pretreated with tunicamycin and then challenged with actinomycin $\mathrm{D} / \mathrm{TNF}$. These results suggest that an $\mathrm{N}$-glycosylated protein is a necessary factor for TNF-induced liver failure in actinomycin D-pretreated mice.

\subsection{Protection of murine hepatocytes from actinomycin $D / T N F$-induced programmed cell death by tunicamycin}

Next we examined whether tunicamycin acted in fact on the hepatocyte, i.e., the dominant target cell of TNF in this model of septic liver failure. Therefore primary liver cell cultures were preincubated with tunicamycin and actinomycin D for $30 \mathrm{~min}$ prior to the addition of TNF. We observed a significant and concentration-dependent protection of hepatocytes from TNF cytotoxicity (Fig. 1A) with a median inhibitory concentration of $8 \pm 2 \mathrm{nM}$ for tunicamycin. The glycosylation inhibitor alone was not toxic towards hepatocytes at concentrations less than $5 \mu \mathrm{M}$. Thus, very low

Table 1

Protection of mice from actinomycin D (ActD)/TNF-induced liver injury by tunicamycin

\begin{tabular}{lccc}
\hline Treatment group & \multicolumn{3}{c}{ Plasma enzymes (U/I) } \\
\cline { 2 - 4 } & ALT & AST & SDH \\
\hline Solvent control & $35 \pm 15$ & $42 \pm 24$ & $17 \pm 3$ \\
TNF & $61 \pm 26$ & $59 \pm 15$ & $18 \pm 33$ \\
ActD & $35 \pm 12$ & $112 \pm 38$ & $19 \pm 7$ \\
ActD/TNF & $4490 \pm 420$ & $2800 \pm 415$ & $2240 \pm 430$ \\
ActD/TNF/tunicamycin & $975 \pm 160^{*}$ & $655 \pm 120^{*}$ & $230 \pm 90^{*}$ \\
\hline
\end{tabular}

$1 \mathrm{mg} / \mathrm{kg}$ tunicamycin was injected intraperitoneally $30 \mathrm{~min}$ before injection of $800 \mu \mathrm{g} / \mathrm{kg}$ ActD and $3 \mu \mathrm{g} / \mathrm{kg}$ TNF. Alanine-aminotransferase (ALT), aspartate-aminotransferase (AST) or sorbitol dehydrogenase (SDH) were determined spectrophotometrically in the plasma samples of blood taken $8 \mathrm{~h}$ after intervention. " $p<0.01 \mathrm{vs.}$ ActD/TNF (Welch test). Data are means $\pm S D$ from six animals. and non-toxic concentrations of tunicamycin were sufficient to protect actinomycin D-sensitized cultured hepatocytes from TNF-induced cell death. The attenuation of TNF toxicity due to the glycosylation inhibitor was not only observed after preincubation, but also when $500 \mathrm{nM}$ tunicamycin was added within $4 \mathrm{~h}$ after TNF (reduction of toxicity by $50 \%$ ). This means that TNF-inducible cytotoxicity is partially reversible following cytokine exposure. These findings are a further indication of the involvement of glycoproteins in actinomycin D/TNF toxicity. This conclusion is also corroborated by our observation that brefeldin $\mathrm{A}$, an inhibitor of glycoprotein translocation from the endoplasmic reticulum to the Golgi protected hepatocytes concentration-dependently from actinomycin $\mathrm{D} / \mathrm{TNF}$ cytotoxicity (median inhibitory concentration: $178 \mu \mathrm{M}$ ).

We tested the influence of tunicamycin on TNF-induced toxicity in the murine fibrosarcoma cell line WEHI-164 clone 13. In this cell line TNF alone (2 $\mathrm{ng} / \mathrm{ml}$ ) induced cell death associated with DNA fragmentation more rapidly than in hepatocytes. Only $3 \mathrm{~h}$ after the addition of TNF, viability was ignificantly reduced and DNA fragmentation was increased 22-fold compared to untreated control WEHI-164 cells. Tunicamycin alone killed WEHI-164 cells with a median effective concentration of $170 \pm 10 \mathrm{nM}$. In the presence of TNF at concentrations that caused 50-90\% cell death $(32-4000 \mathrm{pg} / \mathrm{ml})$ we noticed an additive toxicity of tunicamycin rather than a protection. It may be conjectured that this, completely opposite, sensitivity towards tunicamycin of a transformed cell line represents a killing mechanism different from that in primary organ toxicity of TNF in naive cells, such as the hepatocyte.

Since TNF-induced cytotoxicity in actinomycin Dsensitized liver cells was shown to be preceded by DNA fragmentation and characterized by apoptotic changes (Leist et al., 1994), we examined whether tunicamycin treatment would interfere with these early events of cell death. We measured DNA fragmentation $10 \mathrm{~h}$ after addition of TNF, i.e., at a time point when no significant lactate dehydrogenase release in actinomycin D/TNF-treated cells was detectable. As demonstrated in Fig. 1B, at this time point extensive DNA fragmentation, characterized by a 'ladder pattern' can be documented. This fragmentation was reduced almost to control levels in cells preincubated in the presence of tunicamycin. These findings indicate that tunicamycin-sensitive processes are likely to be involved in early apoptotic changes leading to cell death. The number of hepatocytes with chromatin condensation was quantitated $14 \mathrm{~h}$ after the addition of TNF $(100 \mathrm{ng} / \mathrm{ml})$. On staining with bisbenzimide $\mathrm{H}-33258$ we observed that the percentage of apoptotic nuclei detected in actinomycin D-sensitized hepatocytes (actinomycin D: $12 \pm 5 \%$ ) treated with TNF 
(actinomycin D/TNF: $40 \pm 8 \%$ ) was reduced significantly by pretreatment with $500 \mu \mathrm{M}$ tunicamycin (actinomycin $\mathrm{D} / \mathrm{TNF}^{\circ}+$ tunicamycin: $20 \pm 6 \%$ ). Thus in addition to the inhibition of the early biochemical event, i.e., DNA fragmentation and the late manifestation of toxicity, i.e., lactate dehydrogenase release, tunicamycin also prevented the morphological changes associated with TNF-induced programmed hepatocyte death.

\section{Discussion}

In rodent models of inflammation, transcriptional inhibitors such as D-galactosamine or actinomycin D have been commonly used as sensitizers towards direct or indirect TNF toxicity (Lehmann et al., 1987; Wallach et al., 1988; Wendel, 1990). The pathomechanism induced by TNF involves apoptosis of hepatic parenchymal cells (Leist et al., 1994). Among a large panel of compounds that we screened for the property of preventing cytokine-induced liver cell death, the by far most potent protective agent was the uridyl antibiotic tunicamycin. It did not only prevent cytotoxicity, apoptotic changes and DNA fragmentation in hepatocyte primary cultures exposed to TNF, but also protected mice from TNF-induced hepatic failure. At the nanomolar concentrations of tunicamycin used in this study ribosomal polypeptide synthesis is not significantly affected. However, dolichyl-dependent protein glycosylation is inhibited and typical glycoproteins such as $\alpha_{2}$-macroglobulin, ceruloplasmin or $\alpha_{1}$-protease inhibitor are no longer secreted from liver cells (Yeo et al., 1989). This potent inhibition of hepatocyte apoptosis by tunicamycin indicates that a de novo synthesized glycoprotein is involved in TNF-induced programmed liver cell death.

With hepatocytes treated with tunicamycin only, we observed neither an increased lactate dehydrogenase release, nor a reduced formazan formation from tetrazolium salts nor any morphological signs of tunicamycin-induced cytotoxicity. In contrast to these findings, the antibiotic was toxic to murine fibrosarcoma cells; this is in agreement with previous findings that tunicamycin selectively damages transformed cell lines (Kohno et al., 1979). On the basis of such findings it may be speculated that cotreatment with tunicamycin or related substances may represent a means of increasing the selectivity of TNF for tumor cells.

\section{Acknowledgements}

The perfect technical assistance of S. Otte is gratefully acknowledged. Special thanks go to F. Gantner for experimental support and helpful discussion.

\section{References}

Bergmeyer, H.U., 1984, Methods of enzymatic analysis, 3rd edn. (Verlag Chemie, Weinheim)

Duksin, D. and W.C. Mahoney, 1982. Relationship of the structure and biological activity of the natural homologues of tunicamycin. J. Biol. Chem. 257, 3105.

Elbein, A.D., 1981, The tunicamycins - useful tools for studies on glycoproteins. Trends Biochem. Sci. 6. 219.

Espevik, T. and J. Nissen-Meyer. 1986. A highly sensitive cell line WEHI 164 clone 13, for measuring cytotoxic factor/tumor necrosis factor from human monocytes. J. Immunol. Methods 95, 99.

Kohno, K.. A. Hiragun. H. Mitsui. A. Takatsuki and G. Tamura. 1979, Effect of tunicamycin on cell growth and morphology of nontransformed and transformed cell lines, Agric. Biol. Chem. 43. 1553.

Lehmann V., M.A. Freudenberg, and C. Galanos. 1987. Lethal toxicity of lipopolysaccharide and tumor necrosis factor in normal and D-galactosamine-treated mice. J. Exp. Med. 163, 657.

Leist, M., F. Gantner, I. Bohlinger. H.G. German, G. Tiegs and A. Wendel, 1994. Tumor necrosis factor induced hepaltocyte apoptosis in vivo and in vitro requires transcriptional arrest. J. Immunol. 153. 1778 .

Seglen. P.O., 1973, Preparation of rat liver cells: Enzymatic requirements for tissue dispersion. Exp. Cell. Res. 82. 391.

Tracey. K.J. and A. Cerami. 1993. Tumor necrosis factor. other cytokines and disease. Annu. Rev. Biol. 9. 317.

Waage, A.. 1992. Presence and involtement of TNF in septic shuck. in: Tumor Necrosis Factors: The Molecules and their Emerging Role in Medicine. ed. B. Beutler (Raven Press. New York) p. 275.

Wallach D., H. Holtmann. H. Engelmann, and Y. Nophar. 1988. Sensitization and desensitization to lethal effects of tumor necrosis factor and Il-1. J. Immunol. 1+0. 2994.

Wendel, A., 1990. Biochemical Pharmacology of Intlammatory Liver Injury in Mice. Methods Enzrmol. 186. 675.

Wyllie, A.H., 1980, Glucocorticoid-induced thymocyte apoptosis is associated with endogenous endonuclease activation. Nature 284. 555.

Yeo, T.K., K.T. Yeo and K. Olden. 1989. Accumulation of unglycosylated liver secretory glycoproteins in the rough endoplasmic reticulum, Biochem. Biophys. Res. Commun. 160, 1421. 